\title{
The Relationship between Students Attitude toward Nursing Profession, Achievement Motivation and Academic Performance of Baccalaureate Nursing Students
}

\author{
Abeer Abdallah Ali, Eglal Ahmed A. El-Wahab, \& Sahar Ahmed Abood. \\ Nursing administration dept., Faculty of Nursing- Minia University. \\ Nursing Administration dept, Faculty of Nursing- Cairo University.
}

\begin{abstract}
:
Background: Today's nursing students and future nurses make up the backbone of the health care forces in every country, whose attitudes are influential on other health care providers. Aim of this study: was to investigate the relationship between the attitude toward nursing profession, achievement motivation and academic performance of baccalaureate nursing students. Methodology: A descriptive correlational research design was utilized in the study. Setting: The study was conducted at Faculty of nursing, Minia University. A stratified random sampling procedures were employed for selection of 50\% from the students in each of the four academic years enrolled in Faculty of Nursing, Minia University during the academic year $2012 / 2013$ with total number $(n=385)$. Tools of the study: $\mathbf{1}^{\text {st }}$ tool was attitude toward nursing profession scale to assess students' attitude toward nursing profession. $2^{\text {nd }}$ tool was achievement motivation scale prepared by Khalefa (2006). $3^{\text {rd }}$ tool was an auditing checklist for students' academic performance at the end of the academic year. Results: indicated that the majority of the subjects had positive attitude toward the nursing profession and more than two thirds of them had high achievement motivation. There was statistically significant positive correlation between students' attitude toward nursing profession and their achievement motivation. However, there was no statistically significant correlation between students' attitude toward nursing profession or achievement motivation and their academic performance. Conclusions: Nursing students' attitude tended to be positive towards their future profession. First year students appeared to have more positive attitude and higher level of achievement motivation than other academic years. Recommendations: Nursing educators should strengthen curriculum reform, enhance their own quality as teachers, try new teaching strategies and allow students to experience appropriate clinical environment. Nursing social status in Media needs to be improved.
\end{abstract}

Key words: Attitude, achievement motivation, academic performance, nursing profession.

\section{Introduction:}

Nursing as a profession is an art and a science, placing its focus on the health care of individuals, families, and communities; so that they can attain, maintain, or recover optimum health. The professional nurse views people in their totality in providing a service essential to community health and welfare. Nurses care for individuals of all ages and cultural backgrounds who are healthy and ill in a holistic manner based on the individual's physical, emotional, psychological, intellectual, social, and spiritual needs (Degazon and Shaw, 2007).

Today's nursing students and future nurses make up the backbone of the health care forces in every country, whose attitudes are influential on other health care providers (Namdar et. al., 2008). It appears that the community's points of view on nursing can positively or negatively affect this profession (Miers, Rickaby, and Pollard, 2007). In this line, Saberian (2003) ascribes society's attitude and erroneous and undesirable public opinion as the reasons for students' disappointment in nursing occupation and subsequent adverse effects on the quality of their education and function.
In reality, helping individuals provides nurses with great satisfaction, however, not being able to take active role in diagnosing health problems, the absence of laws of the profession as well as the standardization in the vocational education, and the negative connotations attached to the profession of nursing by the society make nursing students posses negative ideas (Karadag, 2008).

Attitude plays a principal role in guiding human behavior toward achieving goals, awareness of its consequences and effective processing of complex information about living environment. In reality, knowledge, skill, and positive outlook are the characteristics recommended to develop nursing education and new nursing strategies today. To develop nursing education and promote nursing strategies, there is a need for a staff with positive attitude (Cronenwett et. al., 2009).

Motivation plays a valuable part for the explanation of causes of behavior, predicting effects of actions, and directing behavior to achieve goals. It is the internal process that fosters behavior, its energy and direction, these internal processes include one's goals, beliefs, perceptions and expectations. In 
addition, educators are always trying to discover ways to increase retention among students in higher education, and the motivation aspect is one avenue that has been investigated but may need to have more attention as motivation has been positively related to learning outcomes, and positive learning outcomes has been correlated with increased retention in higher education (Jeffreys, 2007).

In this line, one aim of nursing education is to motivate nurses to acquire skills for offering appropriate quality health care services to patients with multiple complex health problems. Achieving this has challenged academic institutions for a long time (Kosgeroglu, Ayranci, and Erkal, 2009). Nursing students need long-term motivation to help others in the future. While there are a number of factors that affect students' performance, one of the most influential is motivation. So paying due attention to the concept of motivation is of great importance in clinical education (Miers, Rickaby, and Pollard, 2007).

Aim of the study:

The current study aimed to investigate the relationship between students' attitude toward nursing profession, achievement motivation and academic performance of baccalaureate nursing students.

\section{SUBJECTS AND METHODS:}

\section{Research design:}

A descriptive correlational research design was utilized in the present study.

\section{Setting:}

This study was carried out at Faculty of nursing, Minia University, that was established in (1995) by Presidential Decree number (184) as Higher Institute of Nursing which was affiliated the Faculty of Medicine then issued Presidential Decree number (200) of year(2000) to turn it to a Faculty of Nursing. The study started in the academic year 1997/1998. The faculty offers baccalaureate nursing program at four levels and an internship program for one calendar year. It consists of two adjacent buildings \& involves six academic departments.

\section{Subjects:}

The study sample included (385) undergraduate baccalaureate nursing students selected by stratified random sampling techniques for proportional allocation of $50 \%$ from the students of each of the four academic years enrolled in Faculty of Nursing, Minia University during the academic year 2012 2013 . This sample divided as (90 student) from the $1^{\text {st }}$ year, (105 student) from the $2^{\text {nd }}$ year, (112 student) from the $3^{\text {rd }}$ year, and (78student) from the $4^{\text {th }}$ year.
Tools of the study:

Data was collected using three instruments as follow:-

1- The Attitude toward Nursing Profession scale. This tool was modified by the researcher from the attitude toward nursing profession scale that was developed by (shaccora, 2002) to assess students' attitude toward nursing profession. It consisted of two parts: the $1^{\text {st }}$ part related to subjects' socio-demographic characteristics of (age, gender, academic year, and place of residence). The $2^{\text {nd }}$ part consisted of sixty statements about nursing profession on a fourpoint likert scale scored as (1) for strongly disagree, (2) for disagree, (3) for agree and (4) strongly agree. The total score of the scale was (240 scores) ranged from (60 - 150) as Negative and from (>150 - 240) as Positive.

It was divided into five domains as follows:

- Persuasion and Self satisfaction with the profession domain. (15 item)

- Professional domain. (11 item)

- Social domain. (16 item)

- Cultural and Educational domain. (10 items)

- Economic domain. (8 items)

2- Achievement motivation scale prepared by Khalefa (2006) and used by (Thrwat, 2009). It was consisted of 50 items divided into five dimensions (10 items) each as follows:

- Sense of responsibility.

- The pursuit of excellence.

- Perseverance.

- Feeling the importance of time.

- Planning for the future.

Students answers was against a five-point likert scale as (1) Does not reflect you at all, (2) reflects you to some extent, (3) moderately reflects you, (4) reflects you to a large extent, and (5) reflects you completely. The total score of the scale was (250 scores) ranged from $(50$ - 150) as Low and from $(>150$ - 250) as High.

3- Auditing checklist developed by the researcher for students' academic performance at the end of the academic year on a five points scale as indicated by the faculty' By lows as follows:

\begin{tabular}{|l|c|c|c|}
\hline $\begin{array}{l}\text { student's } \\
\text { code no. }\end{array}$ & range & $\begin{array}{c}\text { Equivalent } \\
\text { Degree }\end{array}$ & scale range \\
\hline & $>85$ & Excellent & 5 \\
\hline & $75-<85$ & Very good & 4 \\
\hline & $65-<75$ & Good & 3 \\
\hline & $50-<65$ & Pass & 2 \\
\hline & $<50$ & Fail & 1 \\
\hline
\end{tabular}




\section{Validity and Reliability}

The modified attitude toward nursing profession scale was given to a panel of five experts in nursing education field to verify its clarity. The second tool (achievement motivation Scale) was adopted as it is from (Thrwat, 2009) who recommended content validity of that tool in her study. Reliability analysis was performed by using Cronbach's alpha coefficient test that was $(0.73)$ for the attitude scale and (0.89) for the achievement motivation scale, and such results indicate quite high reliability.

\section{Ethical considerations:}

Acceptance to conduct the study was obtained from the ethical committee at the faculty of Nursing, Cairo University. Participants' informed consent was obtained. A covering page was attached to each questionnaire explaining the purpose of the study, and indicating that students' participation in the study was optional, and that their responses would remain confidential.

\section{Pilot study}

A pilot study was conducted on $(10 \%)$ of the study sample $(n=38)$ to estimate the feasibility and practicability of the study tools and to estimate the time needed for filling the two scales that ranged between 20 to 30 minutes as well as to detect any obstacles and problems that may be encountered during data collection time. Data collected from the pilot study were reviewed and used in making the necessary modifications prior to the final application of the study tools and in making reliability analysis. This pilot sample was included in the total of the study sample.

\section{Procedures:}

Official approval for data collection was obtained from the Dean and Vice Dean for Education and Student Affairs at the Nursing Faculty Minia University then the researcher approached the heads of departments in the faculty in order to explain the aim of the study and to obtain their permission to approach the students at the end of clinical days as students would be easily accessed and there would be enough time for them to answer the scales. The scales were submitted to students by the researcher in the faculty's clinical labs. for first year students \& in the clinical settings of Minia university hospitals for the second, third and fourth year students, students were permitted to ask the researcher for any clarification during answering the scales. Data collection procedures started on December 2012 and completed on September 2013 as announcing the students' results was delayed due to unsuitability of the political situation in the country that prevented some of control members from attending to the to complete their work.

\section{Statistical analysis}

Upon completion of data collection, the data were scored, tabulated, and analyzed through data entry and analysis by PC computer using the "Statistical Package for Social Science" (SPSS) version 16. Data were presented using descriptive statistics in the form of (numbers and percentages, mean, and standard deviation). Inferential statistics as (t-test, ANOVA, Chi-square test, and Pearson correlation) was used to identify group differences and association among the study variables.

\section{Results:}

Table (1) : Frequency distribution of the studied sample according to socio-demographic characteristics $(\mathbf{n}=\mathbf{3 8 5})$.

\begin{tabular}{|l|c|c|}
\hline \multicolumn{1}{|c|}{ Socio-demographic characteristics } & No. & \% \\
\hline Age: & 262 & 68.1 \\
\hline $18-<20 \mathrm{yr}$ & 115 & 29.9 \\
\hline $20-22 \mathrm{yr}$ & 8 & 2.0 \\
\hline$>22 \mathrm{yr}$ & 110 & 28.6 \\
\hline Sex: & 275 & 71.4 \\
\hline Male & \multicolumn{3}{l|}{} \\
\hline Female & 269 & 69.9 \\
\hline Place of residence: & 116 & 30.1 \\
\hline Rural & \multicolumn{3}{l|}{} \\
\hline Urban & \multicolumn{3}{|l|}{} \\
\hline
\end{tabular}


Table (2) : Frequency distribution of the studied sample according to their attitude toward nursing profession , achievement motivation, and grade point average $(n=385)$.

\begin{tabular}{|c|c|c|}
\hline Items & No. & $\%$ \\
\hline \multicolumn{3}{|l|}{ Attitude toward nursing profession: } \\
\hline Positive & 353 & 91.7 \\
\hline Negative & 22 & 8.3 \\
\hline \multicolumn{3}{|l|}{ Achievement motivation: } \\
\hline High & 338 & 87.8 \\
\hline Low & 47 & 12.2 \\
\hline \multicolumn{3}{|l|}{ Academic performance: } \\
\hline Fail & 32 & 8.3 \\
\hline Pass & 53 & 13.8 \\
\hline Good & 46 & 11.9 \\
\hline Very good & 143 & 37.1 \\
\hline Excellent & 111 & 28.8 \\
\hline
\end{tabular}

Table (3) : Differences among students in the four academic years regarding mean scores of attitude toward nursing profession $(n=385)$.

\begin{tabular}{|c|c|c|c|c|c|c|c|}
\hline \multirow{2}{*}{ Items } & $\begin{array}{c}1^{\text {st }} \text { year } \\
(n=90)\end{array}$ & $\begin{array}{l}2^{\text {nd }} \text { year } \\
(n=105)\end{array}$ & $\begin{array}{l}3^{\text {rd }} \text { year } \\
(n=112)\end{array}$ & $\begin{array}{c}4^{\text {th }} \text { year } \\
(n=78)\end{array}$ & $\begin{array}{c}\text { Total } \\
(n=385)\end{array}$ & \multirow{2}{*}{$\mathbf{f}$} & \multirow[b]{2}{*}{ p-value } \\
\hline & Mean+ SD & Mean +SD & Mean +SD & Mean +SD & Mean+ SD & & \\
\hline $\begin{array}{l}\text { Self satisfaction and } \\
\text { Persuasion with the } \\
\text { profession }\end{array}$ & $42.13 \pm 9.55$ & $44.66 \pm 9.67$ & $43.41 \pm 9.59$ & $\begin{array}{c}41.87+ \\
8.58\end{array}$ & $43.14 \pm 9.44$ & 1.756 & .155 \\
\hline $\begin{array}{l}\text { Professional aspect } \\
\text { of the profession }\end{array}$ & $33.45 \pm 4.67$ & $34.20 \pm 3.96$ & $32.53 \pm 4.70$ & $\begin{array}{c}32.45 \pm \\
4.58\end{array}$ & $33.18 \pm 4.52$ & $3.424 *$ & .017 \\
\hline $\begin{array}{l}\text { Social aspect of the } \\
\text { profession }\end{array}$ & $52.65 \pm 5.52$ & $53.16 \pm 6.07$ & $51.45 \pm 9.11$ & $\begin{array}{l}51.65 \pm \\
5.06\end{array}$ & $52.24 \pm 6.83$ & 1.435 & .232 \\
\hline $\begin{array}{l}\text { Cultural and } \\
\text { Educational aspect } \\
\text { of the profession }\end{array}$ & $34.73 \pm 3.51$ & $34.49 \pm 3.41$ & $32.43 \pm 5.34$ & $\begin{array}{c}33.45 \pm \\
4.11\end{array}$ & $33.75 \pm 4.30$ & $\begin{array}{c}6.438 * \\
*\end{array}$ & .000 \\
\hline $\begin{array}{l}\text { Economic aspect of } \\
\text { the profession }\end{array}$ & $23.77 \pm 3.42$ & $24.17 \pm 3.34$ & $22.53 \pm 3.58$ & $\begin{array}{l}22.57 \pm \\
2.95\end{array}$ & $23.28 \pm 3.42$ & $\begin{array}{c}6.101 * \\
*\end{array}$ & .000 \\
\hline $\begin{array}{l}\text { Total score of } \\
\text { attitude }\end{array}$ & $\begin{array}{c}186.75+ \\
21.36\end{array}$ & $\begin{array}{c}190.79+ \\
20.30\end{array}$ & $\begin{array}{c}182.27+ \\
25.19\end{array}$ & $\begin{array}{c}182.04+ \\
18.74\end{array}$ & $\begin{array}{c}185.60+ \\
22.02\end{array}$ & $3.631 *$ & .013 \\
\hline
\end{tabular}

significant Diff. at $(\mathrm{p}<0.05)$ level. 
Table (4): Students attitude toward nursing profession according to gender \& residence $(n=385)$.

\begin{tabular}{|c|c|c|c|c|c|c|c|c|}
\hline \multirow[b]{2}{*}{ Items } & \multicolumn{2}{|c|}{$\begin{array}{c}\text { Mean } \pm \text { SD } \\
\text { Gender }\end{array}$} & \multirow{2}{*}{$\mathbf{t}$} & \multirow{2}{*}{$\begin{array}{c}\text { p- } \\
\text { value }\end{array}$} & \multicolumn{2}{|c|}{$\begin{array}{l}\text { Mean } \pm \text { SD } \\
\text { Residence } \\
\end{array}$} & \multirow{2}{*}{$\mathbf{t}$} & \multirow{2}{*}{$\mathbf{p}$-value } \\
\hline & Male & Female & & & Rural & Urban & & \\
\hline $\begin{array}{l}\text { Self satisfaction and } \\
\text { Persuasion with the profession }\end{array}$ & $\begin{array}{l}43.40 \pm \\
9.98\end{array}$ & $\begin{array}{l}43.03 \pm \\
9.22\end{array}$ & .341 & 733 & $\begin{array}{r}43.04 z \\
+9.59\end{array}$ & $\begin{array}{l}43.38 \\
\pm 9.11\end{array}$ & .326 & .745 \\
\hline $\begin{array}{l}\text { Professional aspect of the } \\
\text { profession }\end{array}$ & $\begin{array}{l}33.23 \pm \\
5.10\end{array}$ & $\begin{array}{c}33.17 \pm \\
4.28\end{array}$ & .128 & 898 & $\begin{array}{l}33.19 \\
+4.50\end{array}$ & $\begin{array}{r}33.17 \\
+4.59 \\
\end{array}$ & .049 & .961 \\
\hline Social aspect of the profession & $\begin{array}{c}50.99 \pm \\
6.84\end{array}$ & $\begin{array}{c}52.74 \pm \\
6.78\end{array}$ & $2.28^{*}$ & 023 & $\begin{array}{r}52.24 \\
+7.38 \\
\end{array}$ & $\begin{array}{c}52.24 \pm 5 \\
.39\end{array}$ & .000 & 1.01 \\
\hline $\begin{array}{l}\text { Cultural and Educational } \\
\text { aspect of the profession }\end{array}$ & $\begin{array}{c}32.80 \pm \\
5.02\end{array}$ & $\begin{array}{r}34.13 \\
+3.93 \\
\end{array}$ & $2.75^{* *}$ & 006 & $\begin{array}{r}33.57 \\
+4.31 \\
\end{array}$ & $\begin{array}{r}34.14 \\
+4.28 \\
\end{array}$ & 1.19 & .234 \\
\hline $\begin{array}{l}\text { Economic aspect of the } \\
\text { profession }\end{array}$ & $\begin{array}{l}22.42 \pm \\
3.70\end{array}$ & $\begin{array}{l}23.62 \pm \\
3.25\end{array}$ & $3.13^{* *}$ & 002 & $\begin{array}{r}23.11 \\
+3.51 \\
\end{array}$ & $\begin{array}{c}23.67 \pm \\
3.17\end{array}$ & 1.48 & .140 \\
\hline Total score of attitude & $\begin{array}{c}182.92 \pm \\
23.80\end{array}$ & $\begin{array}{l}186.67 \pm \\
21.21\end{array}$ & 1.51 & 131 & $\begin{array}{c}185.13 \\
\pm \\
22.59\end{array}$ & $\begin{array}{l}186.67 \pm \\
20.68\end{array}$ & .629 & .530 \\
\hline
\end{tabular}

significant at $(\mathrm{p}<0.05)$ level.

Table (5) : Differences among students in the four academic years regarding mean scores of achievement motivation $(\mathbf{n}=\mathbf{3 8 5})$.

\begin{tabular}{|c|c|c|c|c|c|c|c|}
\hline \multirow{2}{*}{ Items } & $\begin{array}{l}1^{\text {st }} \text { year } \\
(n=90)\end{array}$ & $\begin{array}{c}4^{\text {th }} \text { year } \\
(n=78)\end{array}$ & $\begin{array}{l}3^{\text {rd }} \text { level } \\
(n=112)\end{array}$ & $\begin{array}{c}4^{\text {th }} \text { level } \\
(n=78)\end{array}$ & $\begin{array}{c}\text { Total } \\
(\mathrm{n}=\mathbf{3 8 5})\end{array}$ & \multirow[b]{2}{*}{ f } & \multirow[b]{2}{*}{$\mathbf{p}-$ value } \\
\hline & Mean \pm SD & $\underset{\text { SD }}{\operatorname{Mean} \pm}$ & $\begin{array}{c}\text { Mean } \\
+ \text { SD } \\
\end{array}$ & $\begin{array}{c}\text { Mean } \\
+ \text { SD }\end{array}$ & $\underset{\text { SD }}{\operatorname{Mean} \pm}$ & & \\
\hline Sense of responsibility & $37.19 \pm 5.65$ & $\begin{array}{c}36.85 \pm \\
5.49\end{array}$ & $\begin{array}{c}33.97 \pm \\
4.07\end{array}$ & $\begin{array}{l}33.79 \pm \\
5.08\end{array}$ & $\begin{array}{c}35.47 \pm \\
5.29\end{array}$ & 1.756 & .155 \\
\hline $\begin{array}{c}\text { The pursuit of } \\
\text { excellence }\end{array}$ & $39.52 \pm 5.547$ & $\begin{array}{c}37.58 \pm \\
5.38\end{array}$ & $\begin{array}{l}35.45 \pm \\
5.51\end{array}$ & $\begin{array}{c}34.50 \pm \\
5.34\end{array}$ & $\begin{array}{c}36.79 \pm \\
5.74\end{array}$ & $3.424 *$ & .017 \\
\hline Perseverance & $37.31 \pm 6.22$ & $\begin{array}{r}36.73 \\
+5.92 \\
\end{array}$ & $\begin{array}{c}35.419 \pm \\
5.479\end{array}$ & $\begin{array}{r}35.07 \\
+6.187 \\
\end{array}$ & $\begin{array}{r}36.15 \\
+5.969 \\
\end{array}$ & 1.435 & .232 \\
\hline $\begin{array}{l}\text { Feeling the importance } \\
\text { of time }\end{array}$ & $35.70 \pm 7.33$ & $\begin{array}{c}35.40 \pm \\
6.16\end{array}$ & $\begin{array}{c}34.36 \pm \\
6.07\end{array}$ & $\begin{array}{l}34.66 \pm \\
5.85\end{array}$ & $\begin{array}{c}35.02 \pm \\
6.37\end{array}$ & $\begin{array}{c}6.438 * \\
*\end{array}$ & .000 \\
\hline Planning for the future & $36.85 \pm 5.38$ & $\begin{array}{c}35.43 \pm \\
5.19\end{array}$ & $\begin{array}{c}35.92 \pm \\
6.03\end{array}$ & $\begin{array}{c}34.82 \pm \\
5.48\end{array}$ & $\begin{array}{l}35.78 \pm \\
5.57\end{array}$ & $\begin{array}{c}6.101 * \\
*\end{array}$ & .000 \\
\hline Total score of attitude & $\begin{array}{l}185.09 \pm \\
25.48\end{array}$ & $\begin{array}{r}182.00 \\
+22.48 \\
\end{array}$ & $\begin{array}{c}174.84 \pm \\
22.39\end{array}$ & $\begin{array}{c}172.86 \pm \\
20.46\end{array}$ & $\begin{array}{c}178.79 \pm \\
23.24\end{array}$ & $3.631 *$ & .013 \\
\hline
\end{tabular}

$*$ The mean difference is significant at $(\mathrm{p}<0.05)$ level.

** The mean difference is highly significant at $(\mathrm{p}<0.01)$ level. 
Table (6) : students achievement motivation according to gender \& residence $(n=385)$.

\begin{tabular}{|c|c|c|c|c|c|c|c|c|}
\hline \multirow{2}{*}{ Items } & \multicolumn{2}{|c|}{$\begin{array}{c}\text { Mean } \pm \text { SD } \\
\text { Gender }\end{array}$} & \multirow{2}{*}{$\mathbf{t}$} & \multirow{2}{*}{$\mathbf{p}-$ value } & \multicolumn{2}{|c|}{$\begin{array}{c}\text { Mean } \pm \text { SD } \\
\text { Residence }\end{array}$} & \multirow{2}{*}{$\mathbf{t}$} & \multirow{2}{*}{$\mathbf{p}$-value } \\
\hline & Male & Female & & & Rural & Urban & & \\
\hline Sense of responsibility & $35.40 \pm 5.13$ & $\begin{array}{l}35.50 \pm \\
5.35\end{array}$ & .170 & .865 & $\begin{array}{c}35.68 \pm \\
5.33\end{array}$ & $\begin{array}{c}34.97 \pm \\
5.17\end{array}$ & 1.21 & .225 \\
\hline $\begin{array}{l}\text { The pursuit of } \\
\text { excellence }\end{array}$ & $36.00 \pm 5.44$ & $\begin{array}{c}37.10 \pm \\
5.84\end{array}$ & 1.69 & .091 & $\begin{array}{c}36.87 \pm \\
5.748\end{array}$ & $\begin{array}{c}36.59 \pm \\
5.749\end{array}$ & .442 & .658 \\
\hline Perseverance & $36.45 \pm 5.82$ & $\begin{array}{c}36.03 \pm \\
6.03\end{array}$ & .631 & .528 & $\begin{array}{l}36.19 \pm \\
5.96\end{array}$ & $\begin{array}{c}35.17 \pm \\
6.27\end{array}$ & .232 & .817 \\
\hline $\begin{array}{l}\text { Feeling the importance } \\
\text { of time }\end{array}$ & $35.01 \pm 6.14$ & $\begin{array}{c}35.02 \pm \\
6.47\end{array}$ & .018 & .986 & $\begin{array}{c}35.17 \pm \\
6.27\end{array}$ & $\begin{array}{c}34.66 \pm \\
6.60\end{array}$ & .716 & .474 \\
\hline Planning for the future & $35.37 \pm 5.55$ & $\begin{array}{l}35.95 \pm \\
5.58\end{array}$ & .922 & .357 & $\begin{array}{l}35.83 \pm \\
5.40\end{array}$ & $\begin{array}{c}35.68 \pm \\
5.97\end{array}$ & .245 & .807 \\
\hline $\begin{array}{l}\text { Total score of } \\
\text { achievement motivation }\end{array}$ & $\begin{array}{l}177.57 \pm \\
21.85\end{array}$ & $\begin{array}{c}179.27 \pm \\
23.79\end{array}$ & .649 & .517 & $\begin{array}{c}179.37 \pm \\
23.08\end{array}$ & $\begin{array}{r}177.43 \\
+\quad 23.63 \\
\end{array}$ & .629 & .530 \\
\hline
\end{tabular}

$*$ The mean difference is significant at $(\mathrm{p}<0.05)$ level.

** The mean difference is highly significant at $(\mathrm{p}<0.01)$ level.

Table (7) : Correlation between the attitude toward nursing profession and achievement motivation among students of the four academic years $(n=385)$.

\begin{tabular}{|c|c|c|c|c|c|c|c|}
\hline $\begin{array}{l}\text { Achievement motivation } \\
\quad \text { Attitude } \\
\text { toward nursing } \\
\text { profession }\end{array}$ & & $\begin{array}{l}\text { Sense of } \\
\text { responsi } \\
\text { bility }\end{array}$ & $\begin{array}{l}\text { The } \\
\text { pursuit of } \\
\text { excellence }\end{array}$ & $\begin{array}{l}\text { Persever } \\
\text { ance }\end{array}$ & $\begin{array}{l}\text { Feeling the } \\
\text { importance } \\
\text { of time }\end{array}$ & $\begin{array}{l}\text { Planning } \\
\text { for the } \\
\text { future }\end{array}$ & $\begin{array}{l}\text { Total_score } \\
\text { of } \\
\text { achievemen } \\
\text { t motivation }\end{array}$ \\
\hline \multirow{2}{*}{$\begin{array}{l}\text { Self satisfaction and } \\
\text { Persuasion with the } \\
\text { profession }\end{array}$} & $\mathbf{r}$ & $.223^{* *}$ & $.117^{*}$ & $.188^{* *}$ & $.127^{*}$ & .050 & $183^{* *}$ \\
\hline & p & .000 & .022 & .000 & .012 & .326 & .000 \\
\hline \multirow{2}{*}{$\begin{array}{l}\text { Professional aspect of the } \\
\text { profession }\end{array}$} & $\mathbf{r}$ & $.229^{* * *}$ & $.227^{* * *}$ & $.234^{* *}$ & $.121^{*}$ & $.103^{*}$ & $.232^{* *}$ \\
\hline & p & .000 & .000 & .000 & .017 & .043 & .000 \\
\hline \multirow{2}{*}{$\begin{array}{l}\text { Social aspect of the } \\
\text { profession }\end{array}$} & $\mathbf{r}$ & $.244^{* * *}$ & $.218^{* * *}$ & $.247^{* * *}$ & $181^{* *}$ & $.196^{* * *}$ & $.273^{* *}$ \\
\hline & p & .000 & .000 & .000 & .000 & .000 & .000 \\
\hline \multirow{2}{*}{$\begin{array}{l}\text { Cultural and Educational } \\
\text { aspect of the profession }\end{array}$} & $\mathbf{r}$ & $.160^{* *}$ & $.206^{* *}$ & $.219^{* *}$ & $.203^{* *}$ & $.235^{* *}$ & $.264^{* *}$ \\
\hline & p & .002 & .000 & .000 & .000 & .000 & .000 \\
\hline \multirow{2}{*}{$\begin{array}{l}\text { Economic aspect of the } \\
\text { profession }\end{array}$} & $\mathbf{r}$ & $.198^{* * *}$ & $.217^{* * *}$ & $.178^{* * *}$ & $.148^{* * *}$ & $.151^{* * *}$ & $.244^{* * *}$ \\
\hline & p & .000 & .000 & .000 & .004 & .003 & .000 \\
\hline \multirow{2}{*}{ Total score of attitude } & $\mathbf{r}$ & $.280^{* * *}$ & $.238^{* * *}$ & $.276^{* * *}$ & $.198^{* * *}$ & $.173^{* * *}$ & $.301^{* * *}$ \\
\hline & p & .000 & 000 & .000 & .000 & .001 & .000 \\
\hline
\end{tabular}

*. Correlation is significant at the $(\mathrm{p}<0.05)$ level.

**. Correlation is highly significant at the $(\mathrm{p}<0.01)$ level. 
Table (8) : Correlation between the attitude toward nursing profession and the academic performance of the students all over the four academic years $(n=385)$.

\begin{tabular}{|c|c|c|c|c|c|c|}
\hline $\begin{array}{l}\text { Academic performance } \\
\text { Attitude toward } \\
\text { nursing profession Items }\end{array}$ & & $\begin{array}{c}1^{\text {st }} \text { year } \\
(n=90)\end{array}$ & $\begin{array}{l}2^{\text {nd }} \text { year } \\
(n=105)\end{array}$ & $\begin{array}{l}3^{\text {rd }} \text { year } \\
(n=112)\end{array}$ & $\begin{array}{c}4^{\text {th }} \text { year } \\
(n=78)\end{array}$ & $\begin{array}{c}\text { Total } \\
(n=385)\end{array}$ \\
\hline \multirow{2}{*}{$\begin{array}{c}\text { Self satisfaction and Persuasion } \\
\text { with the profession }\end{array}$} & $\mathbf{r}$ & -.072 & -.101 & -.121 & .022 & -.077 \\
\hline & $\mathbf{p}$ & .500 & .304 & .204 & .851 & .130 \\
\hline \multirow{2}{*}{$\begin{array}{c}\text { Professional aspect of the } \\
\text { profession }\end{array}$} & $\mathbf{r}$ & -.012 & -.095 & -.064 & -.159 & $-.110^{*}$ \\
\hline & $\mathbf{p}$ & .909 & .338 & .506 & .165 & .031 \\
\hline \multirow{2}{*}{ Social aspect of the profession } & $\mathbf{r}$ & -.083 & .072 & $-.030-$ & -.006 & -.039 \\
\hline & $\mathbf{p}$ & .437 & .464 & .757 & .956 & .445 \\
\hline \multirow{2}{*}{$\begin{array}{l}\text { Cultural and Educational aspect } \\
\text { of the profession }\end{array}$} & $\mathbf{r}$ & -.154 & .018 & -.070 & .014 & $-.116^{*}$ \\
\hline & $\mathbf{p}$ & .147 & .858 & .460 & .905 & .023 \\
\hline \multirow{2}{*}{$\begin{array}{c}\text { Economic aspect of the } \\
\text { profession }\end{array}$} & $\mathbf{r}$ & .002 & .174 & -.055 & -.034 & $-.035-$ \\
\hline & p & .987 & .076 & .561 & .768 & .489 \\
\hline \multirow{2}{*}{ Total score of attitude } & $\mathbf{r}$ & -.081 & -.020 & -.094 & -.033 & -.099 \\
\hline & $\mathbf{p}$ & .446 & .839 & .322 & .774 & .051 \\
\hline
\end{tabular}

*. Correlation is significant at the $(\mathrm{p}<0.05)$ level.

$* *$. Correlation is highly significant at the $(\mathrm{p}<0.01)$ level.

Table (9) : Correlation between achievement motivation and the academic performance of the students all over the four academic years $(n=385)$.

\begin{tabular}{|c|c|c|c|c|c|c|}
\hline $\begin{array}{rr}\text { Academic } & \text { performance } \\
\text { Achievement } & \text { motivation }\end{array}$ & & $\begin{array}{l}1^{\text {st }} \text { year } \\
(n=90)\end{array}$ & $\begin{array}{l}2^{\text {nd }} \text { year } \\
(n=105)\end{array}$ & $\begin{array}{l}3^{\text {rd }} \text { year } \\
(n=112)\end{array}$ & $\begin{array}{l}4^{\text {th }} \text { year } \\
(n=78)\end{array}$ & $\begin{array}{c}\text { Total } \\
(n=385)\end{array}$ \\
\hline \multirow{2}{*}{ Sense of responsibility } & $\mathbf{r}$ & .061 & .090 & .097 & -.024 & -.040 \\
\hline & p & .567 & .360 & .310 & .837 & .438 \\
\hline \multirow{2}{*}{ The pursuit of excellence } & $\mathbf{r}$ & .083 & .103 & -.004 & .126 & -.047 \\
\hline & $\mathbf{p}$ & .435 & .298 & .964 & .271 & .358 \\
\hline \multirow{2}{*}{ Perseverance } & $\mathbf{r}$ & .127 & -.063 & .103 & -.164 & -.037 \\
\hline & p & .232 & .521 & .281 & .152 & .467 \\
\hline \multirow{2}{*}{ Feeling the importance of time } & $\mathbf{r}$ & -.036 & .095 & -.057 & -.066 & -.036 \\
\hline & $\mathbf{p}$ & .738 & .333 & .548 & .569 & .482 \\
\hline \multirow{2}{*}{ Planning for the future } & $\mathbf{r}$ & -.086 & -.057 & .016 & -.168 & -.081 \\
\hline & $\mathbf{p}$ & .420 & .565 & .866 & .142 & .114 \\
\hline \multirow{2}{*}{$\begin{array}{l}\text { Total score of achievement } \\
\text { motivation }\end{array}$} & $\mathbf{r}$ & .052 & .043 & .020 & -.086 & -.051 \\
\hline & $\mathbf{P}$ & .623 & .663 & .831 & 453 & .315 \\
\hline
\end{tabular}

*. Correlation is significant at the $(\mathrm{p}<0.05)$ level.

**. Correlation is highly significant at the $(\mathrm{p}<0.01)$ level. 
Table (1) : indicated that the majority of subjects $(68.1 \%)$ were between $18-22$ year-old. The majority of them $(71.4 \%)$ were females against $(28.6 \%)$ male students. It also revealed that more than two thirds of the studied sample $(69.9 \%)$ resided in rural areas.

Table (2) : showed that the highest percentage $(91.7 \%)$ of the students had positive attitude toward the nursing profession and more than two thirds $(87.8 \%)$ of them had high achievement motivation. It also revealed that more than one third of the studied sample $(37.1 \%)$ had a very good level of academic performance and less than ten percent $(8.3 \%)$ of them were failed.

Table (3) : revealed that there was statistically significant difference among students all over the four academic years regarding the total score of attitude toward nursing profession especially in the professional aspect of the profession with mean scores (185.60 \pm 22.02 and $33.18 \pm 4.52$ respectively). In addition, there was highly statistical significant difference regarding the" cultural and educational", and "economic" aspect of the profession with mean scores $(33.75+4.30$ and 23.28 \pm 3.42 respectively) especially between the $1^{\text {st }}$ and $4^{\text {th }}$ year students $(34.73 \pm 3.51$ and $33.45 \pm 4.11 ; 23.77 \pm$ 3.42 and $22.57 \pm 2.95$ respectively).

Table (4) : revealed that there was no statistical significant difference between male and female students regarding the total score of attitude toward nursing profession. However, there was highly statistical significant difference favoring to females regarding the" cultural and educational", and the "economic" aspect of the profession with mean scores

(34.13 \pm 3.93 and $23.62+3.25$ respectively). In addition, it also revealed that there was no statistical significant difference among students all over the four academic years in relation to their place of residence.

Table (5) : illustrated that there was highly statistical significant difference among students all over the four academic years regarding the total score of achievement motivation as well as the "sense of responsibility ", and " the pursuit of excellence" domains with mean scores $(178.79 \pm 23.24,35.47 \pm$ 5.29 and $36.79 \pm 5.74$ respectively) especially between the $1^{\text {st }}$ and $4^{\text {th }}$ year students. In addition, there was statistically significant difference regarding the" perseverance" domain with mean scores $(36.15 \pm$ 5.969) higher in the $1^{\text {st }}$ than the $4^{\text {th }}$ year students $(37.31 \pm 6.22$ and $35.07 \pm 6.187$ respectively $)$.

Table (6) : declared that there was no statistical significant difference in mean scores of achievement motivation among students all over the four academic years in relation to their gender (i.e. male, female) or place of residence (i.e. rural, urban).
Table (7) : showed that there was there was highly statistical significant positive correlation between the attitude toward nursing profession and achievement motivation among students all over the four academic years with a score of $(\mathrm{r}=.301$ at $\mathrm{p}$-value $=.000)$.

Table (8) : revealed that there was no statistical significant correlation between students' attitude toward nursing profession and their academic performance at the end of the academic year.

Table (9) : revealed that there was no statistical significant correlation between students' achievement motivation and their academic performance at the end of the academic year.

\section{Discussion:}

The study of nursing prepares and educates students to become professional nurse leaders who can meet the health care needs of diverse populations in an ever-changing and highly technical environment. Nurses are the key members and play the major part of service-providing system in almost all countries. Nursing is also the largest group of health system, possessing considerable potential power that could be influential on the quality of health care services system (Mirzabeigi et al., 2009).

Student motivation has a primary influence on the academic success or failure of college students. Student motivation is a key factor in determining whether college students are successful in their academic careers and, ultimately their professional careers. Motivation is an internal drive within a person to move to action to complete a task. Student motivation encompasses self-regulation, determination, and efficacy. It is important for educators to develop curricula that encourage students to maintain their educational focus and motivation (Parlett, 2012).

Therefore, the present study was conducted to investigate the relationship between students' attitude toward nursing profession, achievement motivation and academic performance of baccalaureate nursing students. Findings of the present study revealed the following:

Concerning the demographic characteristics of the studied sample (Table 1), the present study clarified that the mean age of the students ranged between 18 to 22 years old, the majority of them were females and more than two thirds resided in rural areas.

The attitude of the most nursing students was believed to be negative towards their profession (Rossiter, Bidewel, and Chan, 1998; Al-Omar, 2004; Degazon, and Shaw, 2007; Neilson, and Lauder 2008); however, the present study results were in contrast to this as it found positive attitude toward nursing profession among the majority 
$(91.7 \%)$ of undergraduate nursing students at Faculty of nursing, Minia University, while a minority of them had negative attitude toward nursing profession (Table 2).

These results were in agreement with the finding presented in the study of (Zhang and Petrini, 2008), in which more than half of nursing students held positive attitudes toward the nursing profession, and in (Noruzi, Hajiamini and Ebadi, 2012), which reported that the majority of nursing students had positive attitude toward their profession. These findings were also consistent with the finding achieved by Miligi and Selim, (2013), who reported, the majority of Saudi nursing students had positive attitude toward nursing profession. On the other hand, these findings were not in accordance with the results achieved by Joulaee, Mehrdad, and Bohrani, (2006) who reported $18.3 \%$ negative attitude toward the nursing profession.

In regard to achievement motivation (Table 2), the current study revealed that, more than two thirds $(87.8 \%)$ of the students had high achievement motivation. The level of achievement motivation in this study was consistent with the study conducted by Muldrow, (2008) in which the mean level of achievement motivation was (87.9\%). Also, the previous study's finding was in agreement with the study of (Abesha, 2012), in which the level of achievement motivation was found to be higher or above average.

Regarding the differences in students' attitude toward nursing profession (Table 3), there were statistically significant differences among students of the four academic years in the total score of attitude and in the scores of the "professional", "cultural and educational" and "economic" aspects of the profession with mean scores all higher in the first year students. These findings were consistent with the findings of (Bolan and Grainger, 2009) study in Canada, who uncovered that beginning students held the image that nurses are kind and compassionate, the beliefs that nursing is exciting and that nurses feel good about what they do much more strongly than senior students. While, this result was contrary to the results achieved in the study of (Toth, Dobratz, and Boni, 1998) who found no significant differences in US students' attitudes towards nursing across the years of a nursing program or between nursing students and practicing nurses.

What was unexpected was that, when total scores on the attitude questionnaire were compared among students in relation to their gender \& place of residence (Table4), no statistically significant differences were found between neither male and female nor urban and rural students. However, there were, highly statistical significant differences favoring to female students on some individual domains of attitude scale including the "social", "cultural and educational" and "economic" aspect of the profession.

The previous finding was in accordance with the study of (Zhang and Perini, 2008), which indicated that no significant correlations were found between the students' attitudes towards nursing and their demographic characteristics. Also, it was in line with the study of (AL Thagafi, 2006), in that no significant difference was found in students' attitudes towards nursing profession due to place of residence.

Concerning the differences in achievement motivation (Table 5, 6), the present study uncovered that, regardless of students gender or place of residence, there was highly statistical significant difference in the achievement motivation among students all over the four academic years with mean scores higher in first year students than in other academic years.

For the difference in achievement motivation among the four academic years, the current findings were similar to that of the previous study (Tsige, 2006), which revealed that the first year students of the College of Commerce of Addis Ababa University had high academic achievement motivation.

The findings of this study related to gender differences in achievement motivation were consistent with a previous study on university students (Abesha, 2012), which documented that there were no significant sex differences in achievement motivation. However, the present findings were inconsistent with the findings of (Negasi, 2009), which revealed that male students had significantly higher achievement motivation when compared with their female counterparts.

At last, regarding the relationships between students' attitude toward nursing profession, achievement motivation and their academic performance (Table 7, 8, 9), findings of the present study indicated that, there was highly statistical significant positive correlation between students' attitude toward nursing profession and their achievement motivation. However, a negative correlations were found between both students' attitude toward nursing profession or achievement motivation and their academic performance, but this relation wasn't statistically significant.

These findings were consistent with the findings of previous studies conducted in colleges/univesities (Daniel, 1992; Mulugeta, 1998; Abesha, 2012), which uncovered that undergraduate first year university students had high academic achievement motivation but low academic achievement. And from the other way, the current findings were not in support of many previous studies which documented 
that achievement motivation had a significant and positive effect on the academic achievement of students in higher education institutions

(Caldwell \& Obasi., 2010; Mahyuddin, Elias, \& Noordin., 2009; Turner, Chandler, \& Heffer., 2009; Turner \& Heffer., 2005; Vansteenkiste, 2005; et al., White \& Fogarty, 2001).

Although, there were some studies that attributed the attitude toward nursing profession either individually or in association with variables other than the achievement motivation or the academic performance, there has been no previous study that correlated the attitude of nursing students toward nursing profession with either the achievement motivation or the academic performance, so it was not possible to evaluate the findings of the current study relative to those of other previous studies.

\section{Conclusions:}

In the light of the present findings it was concluded that: The majority of students in Minia faculty of nursing had a positive attitude toward nursing profession and had a high achievement motivation level. Two thirds of students at all levels had "excellent" and "very good" levels of academic performance. Female students were more positively regard the nursing profession than males. First year students appeared to have higher means scores in the attitude toward nursing profession and in achievement motivation than students of the other academic years. There was highly statistical significant positive correlation between students' attitude toward nursing profession and their achievement motivation. However, there was a negative correlation between both the students' attitude toward nursing profession or the achievement motivation and their academic performance, but this relation wasn't statistically significant.

\section{Recommendations:}

Based on important findings of the present study, the following recommendations were suggested:

- Nursing educators should strengthen curriculum reform, enhance their own quality as teachers and allow students to experience appropriate clinical environment to encourage students to maintain their educational performance and motivation throughout their nursing program.

- Establish college support services and academic counseling programs through preceptor ship models who guide, monitor and help nursing students with any problems that limit their academic achievement or motivation.
- It is important to improve nursing social status in Media for promoting the positive attitude of nursing students toward their profession.

- Future similar studies need to be conducted in other nursing colleges to validate the findings of the current study.

\section{References:}

1. Abesha, A., (2012). Effects of Parenting Styles, Academic Self-Efficacy, and Achievement Motivation on the Academic Achievement of University Students in Ethiopia. published doctoral thesis, School of Psychology and Social Science, Faculty of Computing, Health, and Science, Edith Cowan University. Retrieved at January 3, 2014, from ProQuest database.

2. Al-Omar A., Badran (2004). Knowledge, attitudes and intention of high school students towards the nursing profession in Riyadh city, Saudi Arabia. Saudi Med. J; Feb; 25(2); pp. 150- 155.

3. AL Thagafi, H., Hamid, (2006). Change of Attitudes Towards Nursing Profession for a Sample of Saudi Youth Through A Counseling Program. Experimental Study on a sample of students. View at Publisher. View at Google Scholar · View at Scopus.

4. Bolan, C., and Grainger, P. (2009). Students in the BN program - Do their perceptions change? Nurse Education Today, 29 (7); pp. 775-779.

5. Caldwell, T., \& Obasi, E., (2010). Academic performance in African American undergraduates: Effects of cultural mistrust, educational value, and achievement motivation. Journal of Career Development, 36(4), 348-369.

6. Campbell, M., (2007). Motivational systems theory and the academic performance of college students. Journal of College Teaching \& Learning, 4(7), 11-24.

7. Cronenwett L., Sherwood G., Pohl J., Barnsteiner J., Moore S, Sullivan D., (2009). Quality and safety education for advanced nursing practice. Nurse Outlook; 57:338-48.

8. Degazon C., and Shaw H., (2007). Urban high school students' perceptions of nursing as a career choice. J Natl Black Nurses Assoc; Jul, 18 (1):8-13. 
9. Daniel, T., (1992). Personality types and academic achievement motivation as correlates of college academic performance. Unpublished Master Thesis, Addis Ababa University, Addis Ababa, Ethiopia.

10. Jeffreys, M., (2007). Tracking students through program entry, progression, graduation, and licensure: Assessing undergraduate nursing student retention and success. Nurse Education Today Journal, 27, 406-419.

11. Joolaee S., Mehrdad N., Bohrani N.( 2006). A survey on nursing student's opinion toward nursing and reasons for giving it up. Iran J Nurs Res;1:21-8.

12. Karadag G., Güner, I., Çuhadar, D., Uçan, Ö. (2008). Health School Nursing students' selfesteem. Euphrates Health Services Journal, 3 (7), 30-39.

13. Khalefa, M., Abd-El-latief (2006). The psychology of Attitudes. 1st ed., Dar-Gareib puplishers. Ch. 2. pp. 65-129.

14. Kosgeroglu, N., M. B. Acat, U., Ayranci, N. Ozabaci, and S., Erkal, (2009). "An investigation on nursing, midwifery and health care students' learning motivation in Turkey," Nurse Education in Practice, vol. 9, no. 5, pp. 331-339,. View at Publisher. View at Google Scholar . View at Scopus.

15. Mahyuddin, R., Elias, H., \& Noordin, N. (2009). Emotional intelligence, achievement motivation and academic achievement among students of the public and private higher institutions. The International Journal of Diversity in Organistions, Communities and Nations, 9(4), 135-144.

16. Miers, M., Rickaby, C., and Pollard, K., (2007). Career choices in health care: is nursing a special case? A content analysis of survey data. International Journal of Nursing Studies, vol. 44, no. 7, pp. 1196-1209, View at Publisher - View at Google Scholar . View at Scopus.

17. Mirzabeigi G, Salemi S, Sanjari M, Shirazi F., Heidari S., Maleki S.( 2009). Job satisfaction among Iranian Nurses. Hayat; 15:49-59.

18. Muldrow, A., Michelle, (2008). Achievement motivation in African American college students. Published doctoral thesis; ProQuest Dissertations \& Theses (PQDT) pg. n/a.

19. Mulugeta, T., (1998). Locus of control, selfefficacy, and academic achievement motivation as predictors of academic performance of college freshmen: The case of Addis Ababa University. Unpublished Master"s Thesis, Addis Ababa University, Addis Ababa, Ethiopia.
20. Mustofa, A., (2006). Self-efficacy, achievement motivation, attitude and gender difference of students' science performance in Debre-Brhan General Secondary School. Unpublished Master"s Thesis, Addis Ababa University, Addis Ababa, Ethiopia.

21. Namdar H., Arshadi, Mohammad EH, Sahebi HM. (2008). Nursing student's attitude toward mental illnesses. Iran J Nurs Res; 3:15-21.

22. Negasi, H., (2009). The relationship among perception of mathematics classroom,intrinsic motivation, and academic achievement: The case of first year students in faculty of science, Addis Ababa University. Unpublished Master"es Thesis, Addis Ababa University, Addis Ababa, Ethiopia.

23. Neilson G., Lauder W., (2008). What do high academic achieving school pupils really think about a career in nursing: Analysis of the narrative from paradigmatic case interviews. Nurse Education Today; 28; pp. 680-90.

24. Noruzi, A., Koushali, Hajiamini, Z.,and Ebadi, A., (2012). Nursing attitude towards the : Comparison of nursing students' and clinical nurses' attitude toward the nursing profession nursing profession. Iranian Journal of Nursing and Midwifery Research | July-August 2012 | Vol. 17 | Issue 5. www.mui.ac.ir.

25. Parlett, D., (2012). A., Comparison of Associate and Bachelor Degree Nursing Students' Motivation. Published doctoral dissertation, College of social and behavioral sciences, Walden University. Retrieved at December, 2013, from ProQuest database.

26. Rossiter J., Bidewell J,, Chan P., ( 1998). NonEnglish speaking background high school students' attitudes towards the nursing profession. J Adv Nurs; Mar; 27(3); pp. 60413.

27. Saberian, M., (2003). The curriculum of nursing BSc course in the viewpoints of the. J Med Educ; 3:65-70.

28. Shaccora A., (2002). Cognitive Motive and Nursing Students Attitudes toward Nursing Profession and The Relationship of both to The Adjustment to study: published master thesis, Faculty of Education "Psychology department", Islamic University, Gaza.

29. Thrwat R., (2009). Designing a web site for Education Technology course and measuring its effect on achievement motivation for a sample of students at Faculty of Education-Minia University: unpublished master thesis, Faculty of Education, curricula and methods of teaching department, Minia University.

30. Toth J., Dobratz M., and Boni M., (1998). Attitude toward nursing of students earning a second degree and traditional baccalaureate 
students: Are they different? Nurse Outlook, 46; pp. 273-8.

31. Turner, E., Chandler, M., \& Heffer, R., (2009). Influence of parenting styles، achievement motivation, and self-efficacy on academic performance in college students. Journal of College Student Development, 50(3), 337-346.

32. Turner, E., \& Heffer, R., (2005). Parental influences on academic performance and attitudes toward achievement. Retrieved from http://psychology.tamu.edu/ posters/earlturner2005.pdf on 03/09/2008.

33. Tsige, G., (2006). Some correlates of academic performance among students of College of Commerce, Addis Ababa University. The Ethiopian Journal of Education, 26(1), 67-98.

34. Vansteenkiste, M., Zhou, M., Lens, W., \& Soenens, B. (2005). Experiences of autonomy and control among Chinese learners: Vitalizing or immobilizing? Journal of Educational Psychology, 97(3), 468-483.

35. White, C., \& Fogarty, G., (2001). Educational implications of the values held by Australian students. Journal of College Student Retention: Research, Theory, and Practice, 2(3), 253-270.

36. Zhang M., Petrini M., (2008). Factors influencing Chinese undergraduate nursing students' perceptions of the nursing profession. Int Nurs Rev; 55:274-80. 